\section{Neue Methoden für Zukunftswissen}

\author{
Bericht zur Tagung „Planning, \\ Prediction, Scenarios - Using \\ Simulations and Maps“
}

Bonn, 11.-12. Mai 2015

von Martin Deschauer, EA European Academy of Technology and Innovation Assessment, Bad Neuenahr-Ahrweiler

Die Jahrestagung der EA European Academy of Technology and Innovation Assessment stand thematisch ganz im Zeichen von Simulationen, Big Data, Szenario-Analysen und Karten. ${ }^{1}$ Es wurde getestet, welches Potenzial neue sozialwissenschaftliche Methoden besitzen und inwiefern sie ermöglichen, Prognosen und Planungen abzuleiten. Sowohl empirische Fallbeispiele als auch wissenschaftstheoretische Vorträge beleuchteten den Forschungsstand und die Perspektiven neuer Methoden für Planung und Zukunftswissen. Die Beiträge stellten Ergebnisse aus vielfältigen Gegenstandsbereichen vor: Von der Innovationsforschung über Wahlanalysen, Stadtplanung bis hin zu Assessments von Informations- und Wissenssystemen, Universitäts- und Forschungsvorhaben, war ein breites Feld an Themen gegeben.

Die Tagungsorganisatoren etablierten in der Programmplanung neben der wissenschaftlichen Annäherung an Modelle und Simulationen auch einen künstlerischen Zugang zum Thema. Die Kunstausstellung „Places \& Spaces - Mapping Science“ ${ }^{2}$, die mit einer Vernissage am Abend des 11. Mai eröffnet wurde, rückte die Möglichkeiten der Visualisierungen wissenschaftlicher Daten ins Zentrum und betonte damit, dass das ästhetische untrennbar mit dem epistemischen Moment verbunden ist, also wissenschaftlicher Inhalt (Daten) nicht von der Form (Darstellung) $\mathrm{zu}$ unterscheiden ist.

Thematisch ging die Vernissage fließend in die anderen Teile des Programms über. Die vier Sessions, zwei Keynotes und zwei Paneldiskussionen lieferten umfassende Beiträge zur Frage nach Zukunftswissen und Planungshilfen.

\section{Die methodologische Stärke von Simulation}

$\mathrm{Zu}$ Beginn der Tagung wurden in drei Vorträgen sozialwissenschaftliche Simulationen vorgestellt, die verschiedene Zukunftsszenarien und ex-anteEvaluierungen zum Ergebnis hatten. Am Beispiel der irischen Forschungs- und Entwicklungslandschaft zeigten Petra Ahrweiler und Michel Schilperoord (beide EA Bad Neuenahr-Ahrweiler), welche Möglichkeiten der Policy-Planung entstehen, wenn unterschiedliche Szenarien in „Was-wärewenn"-Alternativen durch Computermodelle dargestellt werden können. Nigel Gilbert von der University of Surrey beschrieb den Einsatz von agentenbasierten Modellen (ABM) als gute Möglichkeit, um beispielsweise Konsequenzen aus Policy-Entscheidungen im Vorfeld einzuschätzen. Die mathematischen Modelle, so Gilbert, seien aber keinesfalls eindeutige Vorhersagen, da es sich bei gesellschaftlichen Ereignissen immer um nichtlineare, komplexe Dynamiken handele, die nicht vollständig in mathematischen Modellen abbildbar seien. Ähnlich argumentierte auch Bruce Edmonds von der Manchester Metropolitan University Business School. Er plädierte dafür, nicht immer die gesamte Breite der möglichen Szenarien auszuloten, sondern einige Linien zu verfolgen und diese durch Methoden wie dem ABM auf Mikro-, Mesound Makrolevel zu untersuchen. Die Keynote von John Casti (Wien/Hoboken) spitzte diesen Gedankengang noch einmal zu: Extremereignisse (Casti: $\mathrm{X}$-Events) außerhalb eines Trends ließen sich nicht vorhersagen, seien aber notwendig für Veränderungen und Innovationen. Simulationen seien, so die Einschätzungen der Vortragenden, wenn sie richtig eingesetzt und kontextualisiert werden, durchaus hilfreiche Instrumente zur Einschätzung unterschiedlicher Zukunftsszenarien.

\section{Szenarien}

Bei der Auswahl geeigneter methodischer Elemente für Planungs- und Zukunftswissen plädierte Matthias Weber (AIT Austrian Institute of Technology, Wien) für mehr Diversität. Der stärkere Einbezug der Stakeholder solle dazu führen, dass handlungsleitende, strategische Visionen erarbeitet werden können, die ein Policy-making ermöglichen. 
Im zweiten Vortrag der Session über Szenarien verdeutlichte Andreas Pyka von der Universität Hohenheim, wie durch ABM eine neue Sicht auf Innovationsprozesse etabliert werden könne. Statt von einem technologischen „Spillover“-Effekt auszugehen, der in der wirtschaftswissenschaftlichen Fachliteratur seit langem diskutiert wird, ließen sich Wissensdynamiken viel besser durch Innovationsnetzwerke erklären. Daraus ergäben sich entsprechend neue Konsequenzen für die Strategiegestaltung von Forschung und Entwicklung in öffentlichen und privatwirtschaftlichen Bereichen.

\section{Die Stärke von Big Data und Karten}

Die Vorträge von Katy Börner (Indiana University, Bloomington), Andrea Scharnhorst (Royal Netherlands Academy of Arts and Sciences, Amsterdam) und Panos Argyrakis (Universität Thessaloniki) beschrieben unterschiedliche Big-DataAnsätze im Feld der Wissenschaftsforschung wie z. B. Author-Paper-Networks, Alternativen zum Peer-Review-Verfahren, Visual Analytics of Science und Kollaborationen in EU-Forschungsprogrammen. Die Referenten konnten zeigen, welche Informationen sich aus den größtenteils bereits vorhandenen Daten generieren lassen, sofern der Zugang zu den Daten ermöglicht wird. In diesem Zusammenhang wurden in der Diskussion Fragen zur Offenheit und Verfügbarkeit von Forschungsdaten aufgeworfen. Die Forderung nach Open Data in den Sozialwissenschaften beinhaltet, dass Modelle bis in ihre Einzelheiten nachvollziehbar gemacht werden können. Unter den Schlagworten Open Data/Open Access/Open Science finden sich auch im RRI-Programm und der digitalen Agenda der Europäischen Kommission Forderungen nach einem offenen Umgang mit Daten, die in EU-geförderten Projekten entstanden sind. Leider werde diese Praxis nach Ansicht vieler Tagungsteilnehmenden viel zu wenig verfolgt: Zwischen EU-Idealen und der Forschungsrealität klaffe eine große Lücke. Hier sei ein Kulturwandel notwendig, um das Teilen von Daten selbstverständlicher zu machen. Auf einer ganz praktischen Ebene wurde deutlich, dass das Teilen von Daten im Vorfeld eine Aufbereitung notwendig mache, die von den Projektmitarbeiterinnen und Projektmitarbeitern geleistet werden müsse. Dieser durchaus zeitinten- sive Arbeitsschritt stehe allerdings in Konkurrenz zu den vielfältigen anderen Arbeiten im akademischen Betrieb. Zudem gäbe es dafür, ähnlich wie für eine ausführliche Dokumentation, keinen Platz im Belohnungssystem der Wissenschaft.

Katy Börner, die einen Bogen von ihrem Vortrag zu der, von ihr kuratierten, Ausstellung spannte, erläuterte, wie Visualisierungen als ein analytisches Werkzeug eingesetzt werden können. Auch Karten, ursprünglich ein methodologisches Format der Topographie für die Darstellung des Raums, würden in neue Zusammenhänge gebracht. Sie könnten den Bezug zum euklidischen Raum hinter sich lassen, die Proportionen ändern oder zur Visualisierung vielfältiger Informationszusammenhänge genutzt werden.

Wie in der Diskussion deutlich wurde, geht damit die Notwendigkeit einher, die (nicht immer selbstverständliche) Lesbarkeit von Modellen, Simulationen und Karten zu gewährleisten. Modelle und Visualisierungen hätten das Potenzial, Unbeobachtbares beobachtbar zu machen. Gemeint sei damit, dass Daten nicht für sich selbst sprächen und die Darstellung von Mustern über Visualisierungen neue epistemische Zugänge geben. Trotz (oder gerade wegen) des Potenzials von Visualisierungen müssten verstärkt Lernprozesse forciert werden, die einerseits die Nutzer und Betrachter dazu befähigen, einen kritischen und informierten Umgang zu entwickeln und andererseits den Machern die Wichtigkeit der allgemeinen Lesbarkeit verdeutlichen.

\section{Angewandte Modelle und praktische Planung}

Der Leiter des Instituts für Technikfolgeabschätzung und Systemanalyse (Karlsruhe), Armin Grunwald, beschrieb in seinem Vortrag die grundlegenden Probleme und Hoffnungen der Technikfolgeabschätzung und betonte die Wichtigkeit des Diskurses und der Hinterfragung von Prognosen und Vorhersagen. Anhand einiger Beispiele zeigte Marcus John vom Fraunhofer Institut für Naturwissenschaftlich-technische Trendanalyse (Euskirchen), wie die bibliometrische Forschung Eingang in das methodische Repertoire der Technik-Trendanalyse gefunden habe. Durch eine sog. „Trendarchäologie" würden Publikationsdynamiken erfasst und auf ihre Muster geprüft. Die Erforschung der 
Kollaborationsnetzwerke des University College Dublin gehört für Aoibheann Gibbons zum Portfolio der Organisationsentwicklung. Durch die Darstellungsmöglichkeit ließen sich internationale und außeruniversitäre Kontakte nachvollziehen und auf entsprechende institutionelle Ziele beziehen.

In der Regionalentwicklung können sich durch Siedlungsentwicklungspläne Reserven für Wohn- und Gewerberaum ableiten, wie Hadia Straub vom Regionalverband Ruhr (Essen) in ihrem Vortrag erläuterte. Die Aktivitäten im Verband hätten zum Ziel, die Planung der Landnutzung für die Metropolregion zu gestalten.

\section{Diskussionen}

Am Ende beider Tage wurde jeweils in einer allgemeinen Paneldiskussion mit den Referenten und dem Publikum über die Frage gesprochen, welche Qualitäten ein Modell haben müsse - wann ist es ,gut"? Die scheinbar einfache Frage führte zu weitreichenden Diskussionen und vielfältigen Positionen. In einem Punkt aber waren sich alle einig: Die Qualität bzw. die Güte eines Modells ist immer kontextabhängig - Zweck, Ziel, Adressat und Ausgangsfrage ändern die Einschätzung darüber, ob ein Modell als gut angesehen wird oder nicht.

Simulationen als „Running Theory“ sind zweifach theoriegeleitet, sowohl auf der Ziel- als auch auf der Modellseite. Die Möglichkeiten der Verarbeitung von empirischen Daten, z. B. bei Visualisierungen oder Extrapolationen, erweitern die Vorstellungen eines rein theoretischen Instruments. Die wissenschaftstheoretische Sonderform bringt gleichzeitig eigene Herausforderungen mit sich.

\section{Standards und methodologische Diversität}

Für die Disziplin der Sozialwissenschaften wurden fehlende Standards beklagt, die die Qualität von Modellen verbessern könnten. Dabei wurde aber auch deutlich, dass Strategien der methodologischen Diversität Möglichkeiten bieten, verschiedene Interessen zu bedienen und mehrere Optionen zur Auswahl zu haben. Matthias Weber schlug in seinem Vortrag ein „Concurrent Design Foresight" vor, das unter einer gemeinsamen konzeptuellen Basis ganz verschiedene methodische Ansätze erlauben soll. In dem Vorgehen könnten neben explorativen, prozessualen und strukturellen Modellen auch qualitative und quantitative Varianten eingesetzt werden. Mehr Transparenz im Sinne der Open-Data-Idee würde einer Standardisierung in einigen Modellierungsprozessen vermutlich entgegenkommen. Gleichzeitig beuge der Austausch vor, nicht von den eigenen Modellen ,gefangen“ zu werden, d. h. offen für andere Herangehensweisen zu bleiben. In der Diskussion wurde ein Tradeoff zwischen der Nützlichkeit und der Gefahr eines Modells festgestellt. Diese liegt in der Verführung, den eigenen Modellen zu sehr zu vertrauen und damit in der Konsequenz ihr kommunikatives Potential zu verspielen.

\section{Keine „All purpose“-Modelle}

Es gibt keine „All purpose“-Modelle - d. h. Modelle, die für jeden Kontext passen würden. Verschiedene Ziele können zu unterschiedlichen Bewertungen des gleichen Modells führen. So ist beispielsweise die Modellierung zum wissenschaftlichen Verstehen eines Phänomens nicht zwangsweise für eine politische Handlungsempfehlung brauchbar. Entscheidungsträger übersehen diesen Unterschied oft und sind dann schnell in ihrer Erwartungshaltung enttäuscht. Gerade wenn anstelle von wissenschaftlichen Ergebnissen eher schnelle Antworten mit klaren oder visuell ansprechenden Aussagen erwartet werden, entstehen schnell Missverständnisse, was das Potenzial von Simulationen und Modellen betrifft. Ein ähnliches Problem kann entstehen, wenn Nutzer von Modellen sich zu sehr auf diese verlassen und vergessen, dass es sich um Forschungsobjekte handelt und eben nicht um ausgereifte Anwendungsprodukte. Inwiefern es möglich ist, Vorhersagen durch Modelle zu treffen, wurde ebenfalls an unterschiedlichen Stellen der Tagung diskutiert. Ein Großteil der Aussagen sah eine Vorhersage, also eine konkrete Antwort auf die Fragen, was in der Zukunft passieren wird, als unmöglich an. Armin Grunwald betonte, dass Zukünfte immer eine gemachte Narration seien und einen Autor hätten. Sie erzählten daher implizit mehr über die heutige Zeit, als dass sie einen unverstellten Blickwinkel in die Zukunft hätten. Die „Zutaten“ von Zukünften seien heterogen und unterlägen einer Vielzahl von Einflüssen. Zudem hätten die Debatten über die Zukunft einen Einfluss auf die heutige Welt. 
Es gehe, so eine weitere Teilnehmerin, eher um eine Art der Bewältigung (coping) der Zukunft. Gleichzeitig zeige sich eine Spannung zwischen der Notwendigkeit von Planung und der Undurchsichtigkeit ihrer Auswirkungen.

Ein weiterer kritischer Diskussionsbeitrag betonte den Aspekt der Reduktion von Komplexität, der Modellen innewohne. Sie seien eine Art argumentative „Selbstverteidigung“ der eigenen Position in einer Debatte. In ähnlicher Weise wurden Modelle und Simulationen als Teile von Diskursen und damit eingebettet in soziale Prozesse beschrieben. Sie können also als Argumente in einer wissenschaftlichen Auseinandersetzung beschrieben werden. Ihre Funktionen und Zwecke unterscheiden sich demnach im Hinblick auf den jeweiligen Kontext.

Paul Hoyningen-Huene von der LeibnizUniversität in Hannover machte in seiner Unterscheidung zwischen Vorhersage und Erklärung deutlich, dass beide Begriffe lediglich bei Naturbzw. Kausalgesetzen einen gemeinsamen definitorischen Bereich besitzen. Erklärungen seien ein eher pragmatisches Konzept, das vom Publikum abhänge. Am Beispiel von historischen Ereignissen (narrative Erklärungen) erläuterte er, dass diese nicht für Vorhersagemodelle genutzt werden könnten, da ihre singulären Eigenschaften zu komplex und zu nicht-linear seien.

Einig waren sich die Teilnehmenden in der Besonderheit von Modellen und Simulationen: Sie machen auf Zusammenhänge aufmerksam, die auf andere Art nicht aus den Daten herleitbar wären. Damit stellten Modelle und Simulationen einen speziellen wissenschaftlichen Zugang dar, der sie zu, wie Andrea Scharnhorst es ausdrückte, „Epistemic devices“ mache. Modelle müssten Fragen hervorrufen und zur Auseinandersetzung anregen - ein gutes Modell sei demnach ,a model which makes trouble“.

\section{Anmerkungen}

1) Weitere Informationen zur Tagung: http://www. ea-aw.de/conference-2015

2) Weitere InformationenzurAusstellung: http://www. ea-aw.de/veranstaltungen/kunstausstellungen.html oder unter http://scimaps.org/

\section{\langle\rangle}

\section{Responsible Innovation erreicht Managementforschung}

Bericht von der Konferenz „EURAM'15 - Uncertainty is a Great Opportunity" der European Academy of Management

Warschau, Polen, 17.-20. Juni 2015

von Karsten Bolz, Institut für Technikfolgenabschätzung und Systemanalyse (ITAS), Karlsruhe, Bergische Universität Wuppertal, Schumpeter School of Business and Economics

\section{Die Relevanz der EURAM'15 für die TA-Community}

Die jährlich stattfindende Konferenz der European Academy of Management (EURAM) ist eine der größten Managementkonferenzen in Europa. Doch warum ist sie für die TA Community von Bedeutung? Dieses Jahr stand - neben vielen weiteren spannenden Themen - das Konzept der Responsible Innovation auf der Agenda. Somit war die EURAM'15 die erste große Management und Business Konferenz die diese Thematik explizit behandelte. Während sich die bisherigen Aktivitäten zu dem Konzept auf eine politische und sozioethische Perspektive von akademischen Forschungs- und Entwicklungsprozessen konzentriert (Lettice et al. 2013; Blok/Lemmens 2015), wurde die Rolle von Unternehmen bislang weitgehend ignoriert. Es scheint allerdings besonders wichtig zu sein, Responsible Innovation auch in diesem Kontext zu diskutieren, da (oft) Unternehmen neue Technologien in den Markt einführen, der breiten Gesellschaft verfügbar machen und somit mögliche Folgen herbeiführen. Zudem ist der Punkt an dem Unternehmen neue Technologien in den Markt einführen oftmals Auslöser der öffentlichen Debatte über eben diese. Dies ist möglicherweise aus der Tatsache zu begründen, dass erst zu diesem späten Zeitpunkt die Gesellschaft mit der Neuentwicklung oder neuen Technologie in Berührung kommt bzw. konfrontiert wird (Sutcliffe 2011, S. 8). 